\title{
Characterization of Selected Lactobacillus Strains for Use as Probiotics
}

\author{
Minyu Song ${ }^{1,3}$, Bohyun Yun ${ }^{1}$, Jae-Hak Moon ${ }^{2}$, Dong-June Park ${ }^{4}$, Kwangsei Lim ${ }^{5 *}$, and Sejong Oh ${ }^{1 *}$ \\ ${ }^{1}$ Division of Animal Science, Chonnam National University, Gwangiu 500-757, Korea \\ ${ }^{2}$ Department of Food Science, Chonnam National University, Gwangju 500-757, Korea \\ ${ }^{3}$ Bioresources Inc, Sunchoen 540-843, Korea \\ ${ }^{4}$ Korea Food Research Institute, Sungnam 463-746, Korea \\ ${ }^{5}$ Danone Pulmuone Co. Ltd, Seoul 135-897, Korea
}

\begin{abstract}
The aim of this study was to evaluate the functional properties of lactic acid bacteria from various sources and to identify strains for use as probiotics. Ten Lactobacillus strains were selected and their properties such as bile tolerance, acid resistance, cholesterol assimilation activity, and adherence to HT-29 cells were assessed to determine their potential as probiotics. Lactobacillus sp. JNU 8829, L. casei MB3, L. sakei MA9, L. sakei CH8, and L. acidophilus M23 were found to show full tolerance to the $0.3 \%$ bile acid. All strains without L. acidophilus M23 were the most acid-tolerant strains. After incubating the strains at $\mathrm{pH} 2.5$ for $2 \mathrm{~h}$, their viability decreased by 3 Log cells. Some strains survived at pH 2.5 in the presence of pepsin and $0.3 \%$ bile acid. Lactobacillus sp. JNU 8829, L. acidophilus KU41, L. acidophilus $\mathrm{M} 23$, L. fermentum NS2, L. plantarum M13, and L. plantarum NS3 were found to reduce cholesterol levels by $>50 \%$ in vitro. In the adhesion assay, Lactobacillus sp. JNU 8829, L. casei MB3, L. sakei MA9, and L. sakei CH8 showed higher adhesion activities after $2 \mathrm{~h}$ of co-incubation with the intestinal cells. The results of this comprehensive analysis shows that this new probiotic strain named, Lactobacillus sp. JNU 8829 could be a promising candidate for dairy products.
\end{abstract}

Keywords: Lactobacillus, probiotics, acid and bile acid tolerances, cholesterol

Received July 14, 2015; Revised July 25, 2015; Accepted July 25, 2015

\section{Introduction}

As recently outlined in the Joint Food and Agriculture Organization (FAO)/World Health Organization (WHO) expert consultation on the evaluation of health and nutritional properties of probiotics in food, including milk powder with live lactic acid bacteria (LAB), probiotic strains are defined as live microorganisms that, when consumed in an adequate amount as part of food, provide a health benefit to the host (Servin, 2003). Probiotics are commonly used as feed supplements that exert their beneficial effects by improving the intestinal microbial balance of the host animal. Probiotics used for animals include Lactobacillus, Bifidobacterium, Bacillus, Streptococcus, Pediococcus, and Enterococcus strains (Hyronimus

\footnotetext{
*Corresponding authors: Kwangsei Lim, Danone Pulmuone Co. Ltd, Seoul 135-897, Korea. Tel: +82-2-927-9599, Fax: +82-2927-5199, E-mail: kwangslim@hotmail.com;

Sejong Oh, Division of Animal Science, Chonnam National University, Gwangju 500-757, Korea. Tel: +82-62-530-2116, Fax: +82-62-530-2129, E-mail: soh@chonnam.ac.kr
}

et al., 2000). LAB include native microorganisms residing in the GI tracts of diverse animals. They are believed to play a leading part in the health of the host. The relationship between resident $\mathrm{LAB}$ and the host animal and the role of colonization of the GI tract by LAB in promoting the health of the host have garnered attention (Miyoshi et al., 2006).

LAB such as Lactobacillus and Bifidobacterium spp. have been reported to exert health promoting or 'probiotic' effects in humans and animals (du Toit et al., 1998). Factors such as bile tolerance, which enable a selected strain to survive, grow, and exert therapeutic benefits in the intestinal tract should also form selection criteria for the evaluation of Lactobacillus strains as candidates in the development of probiotic food supplements (Usman and Hosono, 1999).

The probiotic effects of LAB are reported to involve inhibition of pathogenic microorganisms, protection against gastrointestinal diseases, anti-mutagenic and anti-carcinogenic activities, and enhancement of the host immune response (du Toit et al., 1998; Nguyen et al., 2007). The reduction of cholesterol by LAB has also been demonstrated

(9)This is an open access article distributed under the terms of the Creative Commons Attribution Non-Commercial License (http://creativecommons.org/licences/ by-nc/4.0) which permits unrestricted non-commercial use, distribution, and reproduction in any medium, provided the original work is properly cited. 
in humans, mice, and pigs. These cholesterol-lowering effects may be attributed, in part, to the deconjugation of bile acid salts by strains of bacteria that produce the enzyme bile acid salt hydrolase (BSH), as well as shortchain fatty acid fermentation, and cholesterol binding to the bacterial cell wall (Brashears et al., 1998; Pereira and Gibson, 2002). As deconjugated bile acid salts are more readily excreted in the feces than conjugated bile acid salts, bacteria with BSH activity may reduce serum cholesterol by enhancing the excretion of bile acid salts, with a consequent increase in the synthesis of bile acid salts from serum cholesterol, or by decreasing the solubility of cholesterol, and thus reducing its uptake from the gut (Nguyen et al., 2007).

The objectives of this study were to evaluate acid resistance, bile tolerance, adherence to HT-29 cells, and cholesterol-lowering ability of several Lactobacillus strains and to identify potentially useful candidate probiotic Lactobacillus strains.

\section{Materials and Methods}

\section{Selection of Lactobacillus strains}

Table 1 shows the selected Lactobacillus strains. The strains were isolated from kimchi, dairy products, fermented olive and fecal matter from a Korean infant. For the selection of lactobacilli, all samples were cultured on de Man, Rogosa, and Sharpe (MRS) agar (Difco, USA) then acidified to $\mathrm{pH} 5.4$ at $37^{\circ} \mathrm{C}$ for $48 \mathrm{~h}$. All isolates were characterized as Lactobacillus based on Gram staining, catalase test and their ability to change color on the bromocresol purple agar. The final confirmation of their identity was achieved by the 16S rDNA sequence analysis. Ten strains of Lactobacillus were inoculated on plates of MRS agar and incubated for $48 \mathrm{~h}$ at $37^{\circ} \mathrm{C}$. Cultures were maintained in $10 \%$ skim milk with $1 \%$ glucose and $0.3 \%$ yeast extract, and stored at $-80^{\circ} \mathrm{C}$.

\section{Acid and bile tolerance}

Acid and bile tolerance was tested using the method described by Hyronimus et al. (2000). Acid tolerance of cultures were grown in MRS broth at $37^{\circ} \mathrm{C}$ for $18 \mathrm{~h}$ and subcultured in $10 \mathrm{~mL}$ of fresh MRS broth, adjusted to $\mathrm{pH}$ 2.5 with 1,000 units/mL pepsin (Sigma Co., USA). The initial bacterial concentration was $1.0 \times 10^{8} \mathrm{CFU} / \mathrm{mL}$, and samples were incubated for 0 and $2 \mathrm{~h}$ at $37^{\circ} \mathrm{C}$. Cultures $\left(1.0 \times 10^{8} \mathrm{CFU} / \mathrm{mL}\right)$ were serially diluted 10 -fold in 0.05 M sodium phosphate buffer ( $\mathrm{pH} 7.0$ ) to neutralize medium acidity. In the assay of bile tolerance, cells were grown in
Table 1. Selected strains of Lactobacillus used in this study

\begin{tabular}{cc}
\hline \hline Strains & Origin \\
\hline Lactobacillus sp. JNU 8829 & Infant feces \\
Lactobacillus acidophilus KU41 & Infant feces \\
Lactobacillus acidophilus $\mathrm{M} 23$ & Infant feces \\
Lactobacillus brevis CH7 & Kimchi \\
Lactobacillus casei $\mathrm{MB} 3$ & Kimchi \\
Lactobacillus fermentum NS2 & Kimchi \\
Lactobacillus plantarum M13 & Kimchi \\
Lactobacillus plantarum $\mathrm{NS3}$ & Kimchi \\
Lactobacillus sakei CH8 & Fermented olive \\
Lactobacillus sakei MA9 & Fermented olive \\
\hline
\end{tabular}

MRS broth at $37^{\circ} \mathrm{C}$ for $18 \mathrm{~h}$ and then re-cultured in 10 $\mathrm{mL}$ of fresh MRS broth containing $0.3 \%$ (w/v) of oxgall at $37^{\circ} \mathrm{C}$. Bile tolerance was then determined by comparing the final plate count after $24 \mathrm{~h}$ regarding the initial plate count at $0 \mathrm{~h}$. Each experiment was conducted in triplicate.

\section{Cholesterol assimilation}

The cholesterol assimilation assay was performed according to the method described by Buck and Gilliland (1994). The strains were inoculated into MRS broth (polyoxyethanyl cholesteryl sebacate $0.045 \%$ and cysteine $0.05 \%$ ) for $24 \mathrm{~h}$ under anaerobic conditions and then centrifuged $\left(12,000 \mathrm{~g}, 10 \mathrm{~min}, 4^{\circ} \mathrm{C}\right)$. The supernatant was recovered, and the amount of cholesterol remaining in it was determined. The cholesterol content of the supernatant was determined using a modified colorimetric method as previously described by Rudel and Morris (1973). A total of $0.5 \mathrm{~mL}$ supernatant fluid was collected and was mixed with $2 \mathrm{~mL} \mathrm{KOH}(50 \%$, w/v) and $3 \mathrm{~mL} 97 \%$ ethanol and then reacted in a water bath at $60^{\circ} \mathrm{C}$ for $5 \mathrm{~min}$. After cooling, the cells were added to $5 \mathrm{~mL}$ hexane and mixed. A 3 $\mathrm{mL}$ aliquot of distilled water was added, mixed, and clean tubes were allowed to stand for $15 \mathrm{~min}$ at room temperature to allow for phase separation. A $2.5 \mathrm{~mL}$ aliquot of hexane layer fluid was transferred to a new tube, and the hexane was evaporated under nitrogen gas. After the remaining solution was treated with $4 \mathrm{~mL}$ of o-phthalaldehyde reagent $(0.5 \mathrm{mg}$ o-phthalaldehyde/glacial acetic acid $1 \mathrm{~mL}$ ), $2 \mathrm{~mL}$ of sulfuric acid was added and reacted for $10 \mathrm{~min}$. The results were monitored by measuring absorbance with a microplate reader (Synergy HT, Bio-Tek, USA) at $550 \mathrm{~nm}$. Each experiment was conducted in triplicate.

(Cholesterol added)

Cholesterol reduction $(\%)=\frac{-(\text { Cholesterol left })}{\text { Cholesterol added }} \times 100$ 


\section{Adhesion assay}

Assays to determine adhesion were performed using the method of Kim et al. (2008) with slight modifications. The monolayers of HT-29 cells (KCTC, Korea) were washed five times in PBS and overlaid with $0.5 \mathrm{~mL}$ of RPMI 1640 medium (Gibco BRL, USA). A total of $1.0 \times 10^{9} \mathrm{CFU} /$ $\mathrm{mL}$ of strains in antibiotic-free medium were mixed, inoculated to each well, and then incubated for 0 and $24 \mathrm{~h}$ at $37^{\circ} \mathrm{C}$. The monolayer cells were washed 3 times in PBS to remove any unattached bacteria. The adherent cells were released from well plates using $0.2 \%$ trypsin-EDTA and incubated for $5 \mathrm{~min}$ at $37^{\circ} \mathrm{C}$ in $5 \% \mathrm{CO}_{2}-95 \%$ air atmosphere. The number of viable cells was determined using the spread plate method on MRS agar with incubation at $37^{\circ} \mathrm{C}$ for $48 \mathrm{~h}$. Each experiment was conducted in triplicate.

\section{Statistical analysis}

All values are expressed as mean \pm standard error (SE). Data was analyzed by one-way ANOVA using SPSS ver. 18.0 (SPSS Inc., USA). Differences between groups were assessed using Duncan's multiple range test. Statistical significance was considered at $p<0.05$.

\section{Results and Discussion}

\section{Acid and bile tolerance}

Similar $\mathrm{pH}$ tolerance was observed in all strains examined for significant differences in cell viabilities for $2 \mathrm{~h}$, at low $\mathrm{pH}$. All strains showed tolerance to $\mathrm{pH} 2.5$ for $2 \mathrm{~h}$ despite variations in the degree of viability (Table 2 ). The nine strains without L. acidophilus M23 were most acidtolerant strains after incubation at $\mathrm{pH} 2.5$ for $2 \mathrm{~h}$. Out of the nine cells, eight of them decreased by $3 \log$ cells, but one L. acidophilus M23 strain showed decrease by $4.1 \mathrm{log}$ cells. As some strains have numerous acid-shock proteins that promote survival, these are capable of surviving exposure to extreme acidic environments (Merrell and Camilli, 2002). These results are in agreement with other studies which demonstrate that Lactobacillus strains remain viable when exposed to $\mathrm{pH}$ values of 2.5-4.0, but exhibit loss of viability at lower $\mathrm{pH}$ values (du Toit et al., 1998; Dunne et al., 2001; Jacobsen et al., 1999; Liong and Shah, 2005; Maragkoudakis et al., 2006). The term 'probiotic' means 'for life', and describes microorganisms (in most cases, bacteria) that survive passage through the gastrointestinal tract and have beneficial effects on the host (Wang et al., 2012). Acid-tolerant strains have an advantage in surviving in the low $\mathrm{pH}$ conditions of the
Table 2. Survival of isolated strains after $2 \mathrm{~h}$ in modified MRS broth

\begin{tabular}{ccc}
\hline \hline \multirow{2}{*}{ Strains } & \multicolumn{2}{c}{ Pepsin at pH $2.5(\mathrm{CFU} / \mathrm{mL})$} \\
\cline { 2 - 3 } & $0 \mathrm{~h}$ & $2 \mathrm{~h}$ \\
\hline Lactobacillus sp. JNU 8829 & $2.30 \times 10^{8} \pm 0.07$ & $1.76 \times 10^{6} \pm 0.10$ \\
Lactobacillus acidophilus $\mathrm{KU} 41$ & $2.20 \times 10^{8} \pm 0.01$ & $1.70 \times 10^{7} \pm 0.01$ \\
Lactobacillus acidophilus $\mathrm{M} 23$ & $1.60 \times 10^{8} \pm 0.04$ & $1.27 \times 10^{4} \pm 0.02$ \\
Lactobacillus brevis $\mathrm{CH} 7$ & $2.00 \times 10^{8} \pm 0.03$ & $5.00 \times 10^{7} \pm 0.08$ \\
Lactobacillus casei MB3 & $1.24 \times 10^{7} \pm 0.02$ & $4.50 \times 10^{5} \pm 0.36$ \\
Lactobacillus fermentum $\mathrm{NS} 2$ & $8.15 \times 10^{7} \pm 0.04$ & $1.29 \times 10^{6} \pm 0.16$ \\
Lactobacillus plantarum M13 & $3.53 \times 10^{8} \pm 0.04$ & $2.00 \times 10^{7} \pm 0.22$ \\
Lactobacillus plantarum NS3 & $3.90 \times 10^{7} \pm 0.02$ & $7.70 \times 10^{5} \pm 0.28$ \\
Lactobacillus sakei CH8 & $2.00 \times 10^{7} \pm 0.03$ & $1.90 \times 10^{8} \pm 0.04$ \\
Lactobacillus sakei MA9 & $1.63 \times 10^{7} \pm 0.07$ & $1.50 \times 10^{5} \pm 0.15$ \\
\hline
\end{tabular}

Table 3. Effect of $0.3 \%$ bile concentration on the viability of isolated strains

\begin{tabular}{ccc}
\hline \hline \multirow{2}{*}{ Strains } & \multicolumn{2}{c}{$0.3 \%$ oxgall $(\mathrm{CFU} / \mathrm{mL})$} \\
\cline { 2 - 3 } & $0 \mathrm{~h}$ & $24 \mathrm{~h}$ \\
\hline Lactobacillus sp. JNU 8829 & $2.18 \times 10^{6} \pm 0.02$ & $4.45 \times 10^{6} \pm 0.03$ \\
Lactobacillus acidophilus KU41 & $2.28 \times 10^{6} \pm 0.01$ & $3.46 \times 10^{5} \pm 0.33$ \\
Lactobacillus acidophilus M23 & $1.07 \times 10^{6} \pm 0.01$ & $3.87 \times 10^{6} \pm 0.01$ \\
Lactobacillus brevis CH7 & $6.32 \times 10^{6} \pm 0.06$ & $1.08 \times 10^{7} \pm 0.01$ \\
Lactobacillus casei MB3 & $7.84 \times 10^{6} \pm 0.02$ & $6.65 \times 10^{8} \pm 0.11$ \\
Lactobacillus fermentum NS2 & $1.36 \times 10^{6} \pm 0.02$ & $6.90 \times 10^{3} \pm 0.01$ \\
Lactobacillus plantarum M13 & $7.98 \times 10^{5} \pm 0.02$ & $7.48 \times 10^{2} \pm 0.02$ \\
Lactobacillus plantarum NS3 & $7.83 \times 10^{5} \pm 0.02$ & $3.09 \times 10^{3} \pm 0.08$ \\
Lactobacillus sakei CH8 & $7.83 \times 10^{5} \pm 0.01$ & $1.63 \times 10^{7} \pm 0.05$ \\
Lactobacillus sakei MA9 & $6.94 \times 10^{6} \pm 0.21$ & $4.20 \times 10^{8} \pm 0.05$ \\
\hline
\end{tabular}

stomach (as low as pH 2.0), where hydrochloric and gastric acids are secreted (du Toit et al., 1998).

Lactic acid bacteria were isolated and analyzed for their probiotic properties, including acid and bile tolerance. The effects of oxgall on the growth of selected strains revealed Lactobacillus sp. JNU 8829, L. acidophilus M23, L. brevis $\mathrm{CH} 7$, L. casei $\mathrm{MB} 3$, L. sakei $\mathrm{CH} 8$ and L. sakei MA9 to all have full tolerance to $0.3 \%$ bile acid (Table 3 ). These results indicate that bile acid at $0.3 \%$ does not affect the viability of these 4 strains and that all isolates could grow in the presence of $0.3 \%$ bile. In general, the physiological concentration of human bile ranges from $0.3 \%$ to $0.5 \%$ (Dunne et al., 2001). Therefore, resistance to bile acid is an important characteristic that enables Lactobacillus to survive, grow, and remain active in the small intestine (du Toit et al., 1998; Hyronimus et al., 2000). Most studies have reported that the majority of strains survived well under such conditions, suggesting a possible recovery of the initial cell number during passage through the small intestine (Charteris et al., 1998; du Toit et al., 1998; Jacobsen et al., 1999). Chou and Weimer (1999) reported that although such studies have major differences 
species in design, they all show that acid and bile acid have separate and combined effects on the bacterial growth. Therefore, the acid and bile tolerance demonstrated by the LAB studies here suggest that these strains are likely resistant to stomach and intestinal conditions.

\section{Cholesterol assimilation}

The cholesterol assimilation abilities of selected strains of Lactobacillus were investigated (Fig. 1). Lactobacillus sp. JNU 8829, L. acidophilus KU41, L. acidophilus M23, L. fermentum NS2, L. plantarum M13, and L. plantarum NS3 were found to reduce cholesterol levels by $>50 \%$ in the in vitro test. Lactobacillus sakei MA9 and L. sakei CH8 found to decrease cholesterol by $30 \%$, whereas $L$. brevis $\mathrm{CH} 7$ and $L$. casei $\mathrm{MB} 3$ decreased cholesterol slightly below $30 \%$. These results suggest that selected strains were able to remove cholesterol in vitro by inhibiting the formation of cholesterol micelles with bile acids. Cholesterol assimilation by LAB in the gastrointestinal tract would enable the decrease of cholesterol absorption by enterocytes, promoting the excretion of the cholesterol from the host. Noh et al. (1997) suggested that L. acidophilus ATCC 43121 shown resistant greater resistance to lysis by sonication when grown in the presence of cholesterol micelles and bile acid salts. They concluded that this resistance may be due to cholesterol assimilation into the cellular membrane, resulting in sturdier bacterial cells (Noh et al., 1997). Further, Kumar (2012) reported that incorporation of cholesterol micelles by probiotic bacterial cells in the gut may explain the hypocholesterolemic effects exerted by these bacteria. In addition, Nilakhe and Sapre (2015) reported that in vivo hypocholesterolemic ability may be due to cholesterol assimilation by L. acidophilus cells, or attachment of cholesterol to their surface. Therefore, screening for cholesterol-lowering properties in vitro has become an important criterion in the selection of bacterial strains for in vivo probiotic investigations.

\section{Adhesion assay}

Adherence of different bacteria has been studied using eukaryotic cell culture as an in vitro model of the human intestinal mucosa (Lehto and Salminen, 1997). Adhesion and colonization at the intestinal surface may be important prerequisites for probiotic strains to exert beneficial effects in the large intestine (Lim, 2014). The selected strains of Lactobacillus were similarly studied for adhesion to HT-29 cells (Fig. 2). In our study, Lactobacillus sp. JNU 8829, L. acidophilus M23, L. casei MB3, L. sakei CH8, L. sakei MA9 showed higher adhesion abilities than other strains. The viable cell count of Lactobacillus sp. JNU 8829 was lower than that of $L$. casei MB3, but, compared with results of Kim et al. (2008), adhesion of Lactobacillus sp. JNU 8829 showed higher cell viability. Kim et al. (2007) reported that the main criterion in the evaluation of probiotic strains is their ability to adhere to the intestinal epithelial cell line. Therefore, adhesion of the probiotic strains to the intestinal mucosa is considered a prerequisite for successful colonization, and is crucial for antagonistic activity against enteric bacterial pathogens (Ouwehand et al., 1999).

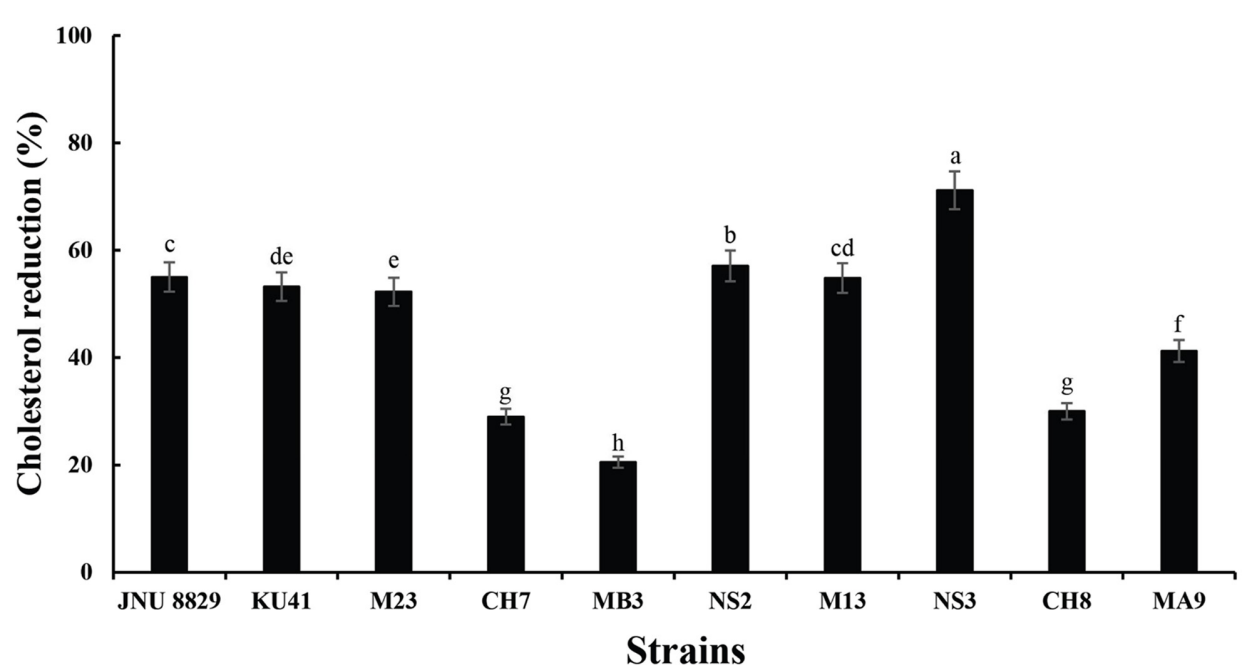

Fig. 1. Reduction of cholesterol by isolated strains in vitro. JNU 8829, Lactobacillus sp.; KU41, L. acidophilus; M23, L. acidophilus; CH7, L. brevis; MB3, L. casei; NS2, L. fermentum; M13, L. plantarum; NS3, L. plantarum; CH8, L. sakei; and L. sakei MA9. Each value is expressed as mean \pm SE. Values with different letters are significantly different $(p<0.05)$. 


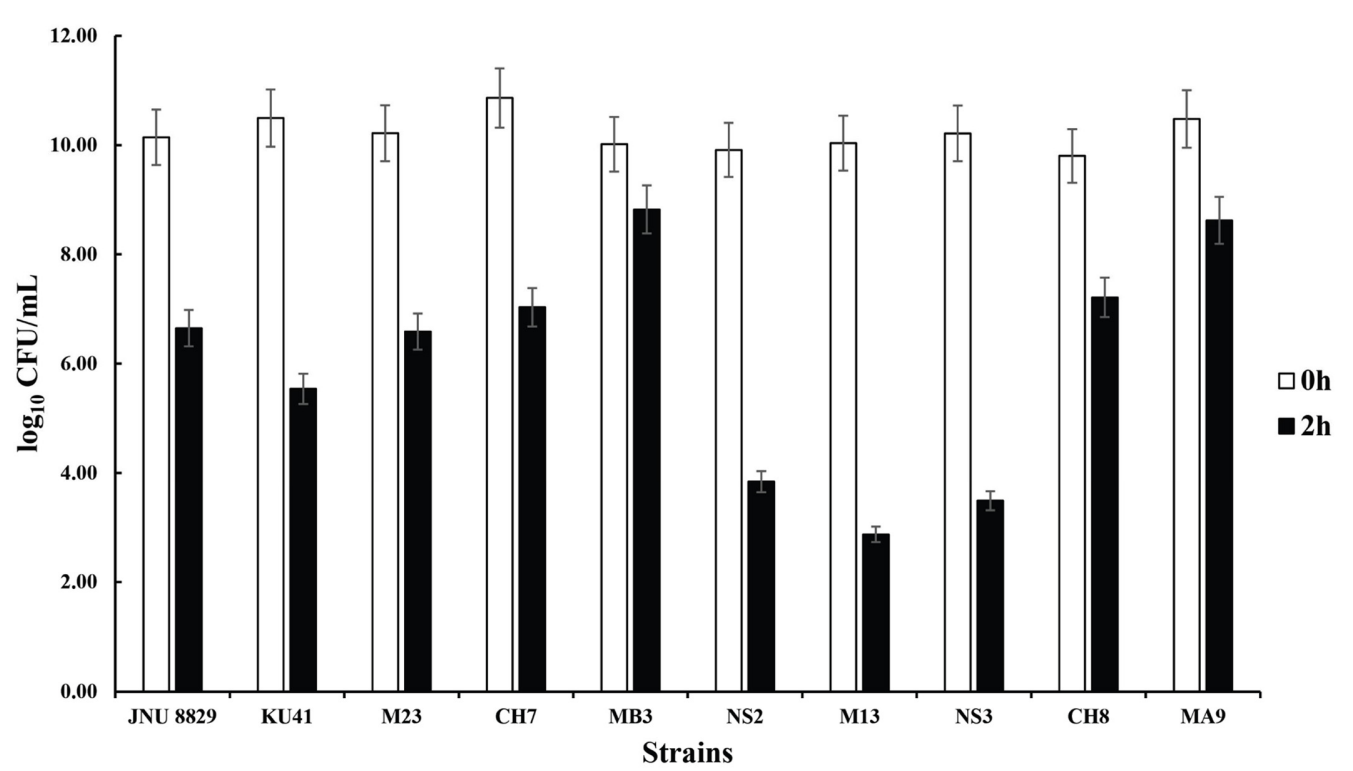

Fig. 2. Adherence of selected Lactobacillus strains to HT-29 cells. JNU 8829, Lactobacillus sp.; KU41, L. acidophilus; M23, L. acidophilus; CH7, L. brevis; MB3, L. casei; NS2, L. fermentum; M13, L. plantarum; NS3, L. plantarum; CH8, L. sakei; and L. sakei MA9. Each value is expressed as mean \pm SE.

\section{Conclusion}

This study was aimed evaluating, in vitro, several probiotic properties of selected LAB strains; namely their acid resistance, bile acid tolerance, cholesterol assimilation activity, and adhesion to cells. The strains evaluated demonstrated sufficient acid and bile tolerance to be resistant to physiological stomach and intestinal conditions, making them potentially useful candidates for the development of probiotics. Cholesterol assimilation by the five strains Lactobacillus sp. JNU 8829, L. acidophilus KU41, L. acidophilus M23, L. fermentum NS2, L. plantarum M13, and L. plantarum NS3 ranged from 52.25 to $71.16 \%$. Lactobacillus sp. JNU 8829, L. fermentum NS2, and L. plantarum NS3 were found to have the highest levels of cholesterol assimilation. These selected Lactobacillus strains also showed adhesive abilities. The Lactobacillus sp. JNU 8829 strain was found to have the most potential effectiveness as a probiotic based on its acid and bile tolerance, cholesterol-lowering effects and adhesion to intestinal cells. Therefore, this strain would make a good candidate for further investigation through in vivo studies to determine its potential health benefits.

\section{Acknowledgements}

This study was supported by High Value-added Food Technology Development Program funded by the Ministry of Agriculture, Food and Rural Affairs (MAFRA) (A20120447) and Technology Commercialization Program funded by INOPOLIS Foundation, the Ministry of Education, Science and Technology (APPL-201502-006141).

\section{References}

1. Brashears, M. M., Gilliland, S. E., and Buck, L. M. (1998) Bile salt deconjugation and cholesterol removal from media by Lactobacillus casei. J. Dairy Sci. 81, 2103-2110.

2. Buck, L. M. and Gilliland, S. E. (1994) Comparison of freshly isolated strains of Lactobacillus acidophilus of human intestinal origin for ability to assimilate cholesterol during growth. J. Dairy Sci. 77, 2925-2933.

3. Charteris, W. P., Kelly, P. M., Morelli, L., and Collins, J. K. (1998) Antibiotic susceptibility of potentially probiotic Bifidobacterium isolates from the human gastrointestinal tract. Lett. Appl. Microbiol. 26, 333-337.

4. Chou, L. S. and Weimer, B. (1999) Isolation and characterization of acid- and bile-tolerant isolates from strains of Lactobacillus acidophilus. J. Dairy Sci. 82, 23-31.

5. du Toit, M., Franz, C. M., Dicks, L. M., Schillinger, U., Haberer, P., Warlies, B., Ahrensc, F., and Holzapfela, W. H. (1998) Characterisation and selection of probiotic lactobacilli for a preliminary minipig feeding trial and their effect on serum cholesterol levels, faeces $\mathrm{pH}$ and faeces moisture content. Int. J. Food Microbiol. 40, 93-104.

6. Dunne, C., O’Mahony, L., Murphy, L., Thornton, G., Morrissey, D., O’Halloran, S., Feeney, M., Flynn, S., Fitzgerald, G., Daly, C., Kiely, B., O’Sullivan, G. C., Shanahan, F., and Collins, J. K. (2001) In vitro selection criteria for probiotic bac- 
teria of human origin: Correlation with in vivo findings. Am. J. Clin. Nutr. 73, 386S-392S.

7. Hyronimus, B., Le Marrec, C., Sassi, A. H., and Deschamps, A. (2000) Acid and bile tolerance of spore-forming lactic acid bacteria. Int. J. Food Microbiol. 61, 193-197.

8. Jacobsen, C. N., Roesnfeldt Nielsen, V., Hayford, A. E., Moller, P. L., Michaelsen, K. F., Paerregaard, A., Sandström, B., Tvede, M., and Jacobsen, M. (1999) Screening of probiotic activities of forty-seven strains of Lactobacillus spp. by in vitro techniques and evaluation of the colonization ability of five selected strains in humans. Appl. Environ. Microbiol. 65, 4949-4956.

9. Kim, P. I., Jung, M. Y., Chang, Y. H., Kim, S., Kim, S. J., and Park, Y. H. (2007) Probiotic properties of Lactobacillus and Bifidobacterium strains isolated from porcine gastrointestinal tract. Appl. Microbiol. Biotechnol. 74, 1103-1111.

10. Kim, S. J., Cho, S. Y., Kim, S. H., Song, O. J., Shin, I. S., Cha, D. S., and Park, H. J. (2008) Effect of microencapsulation on viability and other characteristics in Lactobacillus acidophilus ATCC 43121. LWT-Food Sci. Technol. 41, 493-500.

11. Kumar, R., Grover, S., and Batish, V. K. (2012) Bile salt hydrolase activity screening of lactobacilli in vitro selection of indigenous Lactobacillus strains with potential bile salt hydrolysing and cholesterol-lowering ability. Probiotics Antimicro. Prot. 4, 162-172.

12. Lehto, E. M. and Salminen, S. J. (1997) Inhibition of Salmonella typhimurium adhesion to Caco-2 cell cultures by Lactobacillus strain GG spent culture supernate: Only a pH effect? FEMS Immunol. Med. Microbiol. 18, 125-132.

13. Lim, S. M. (2014) Antimutagenicity activity of the putative probiotic strain Lactobacillus paracasei subsp. tolerans JG22 isolated from pepper leaves Jangajji. Food Sci. Biotechnol. 23, 141-150.

14. Liong, M. T. and Shah, N. P. (2005) Acid and bile tolerance and cholesterol removal ability of lactobacilli strains. J. Dairy Sci. 88, 55-66.

15. Maragkoudakis, P. A., Zoumpopoulou, G., Miaris, C., Kalantzopoulos, G., Pot, B., and Tsakalidou, E. (2006) Probiotic po- tential of Lactobacillus strains isolated from dairy products. Int. Dairy J. 16, 189-199.

16. Merrell, D. S. and Camilli, A. (2002) Acid tolerance of gastrointestinal pathogens. Curr. Opin. Microbiol. 5, 51-55.

17. Miyoshi, Y., Okada, S., Uchimura, T., and Satoh, E. (2006) A mucus adhesion promoting protein, MapA, mediates the adhesion of Lactobacillus reuteri to Caco-2 human intestinal epithelial cells. Biosci. Biotechnol. Biochem. 70, 1622-1628.

18. Nguyen, T. D. T., Kang, J. H., and Lee, M. S. (2007) Characterization of Lactobacillus plantarum $\mathrm{PH} 04$, a potential probiotic bacterium with cholesterol-lowering effects. Int. J. Food Microbiol. 113, 358-361.

19. Nilakhe, S. and Sapre, V. (2015) Cholesterol assimilation by intestinal Lactobacilus acidophilus. Res. J. Chem. Environ. 19, 10-14.

20. Noh, D. O., Kim, S. H., and Gilliland, S. E. (1997) Incorporation of cholesterol into the celluar membrane of Lactobacillus acidophilus ATCC 43121. J. Dairy Sci. 80, 3107-3113.

21. Ouwehand, A. C., Kirjavainen, P. V., Shortt, C., and Salminen, S. (1999) Probiotics: Mechanisms and established effects. Int. Dairy J. 9, 43-52.

22. Pereira, D. I. and Gibson, G. R. (2002) Cholesterol assimilation by lactic acid bacteria and bifidobacteria isolated from the human gut. Appl. Environ. Microbiol. 68, 4689-4693.

23. Rudel, L. L. and Morris, M. D. (1973) Determination of cholesterol using o-phthalaldehyde. J. Lipid Res. 14, 364-366

24. Servin, A. L. and Coconnier, M. H. (2003) Adhesion of probiotic strains to the intestinal mucosa and interaction with pathogens. Best Pract. Res. Clin. Gastroenterol. 17, 741-754.

25. Usman, and Hosono, A. (1999) Bile tolerance, taurocholate deconjugation, and binding of cholesterol by Lactobacillus gasseri strains. J. Dairy Sci. 82, 243-248.

26. Wang, J., Zhang, H., Chen, X., Chen, Y., Menghebilige, and Bao, Q. (2012) Selection of potential probiotic lactobacilli for cholesterol-lowering properties and their effect on cholesterol metabolism in rats fed a high-lipid diet. J. Dairy Sci. 95, 16451654. 